\title{
O Processo de enfermagem sob a ótica do acadêmico de uma faculdade privada de Rondônia
}

\author{
The nursing process under the opinion of the academic of a private faculty of Rondônia \\ El proceso de enfermería bajo la óptica del académico de una facultad privada de \\ Rondonia
}

Jaqueline Michele Santos Barbosa ${ }^{1 *}$, Olga do Nascimento ${ }^{1}$, Ivana Annely Cortez Fonseca ${ }^{1}$.

\begin{abstract}
RESUMO
Objetivo: Descrever o significado do Processo de Enfermagem para os acadêmicos de Enfermagem de uma faculdade privada de Rondônia. Métodos: Trata-se de estudo descritivo, exploratório com abordagem qualitativa. A população pesquisada correspondeu aos acadêmicos do quarto ao décimo período do curso de Enfermagem. A pesquisa foi realizada através da aplicação de um questionário contendo 06 (seis) perguntas abertas. Os dados obtidos foram analisados por método que consiste em leituras repetidas das respostas dos entrevistados, sua separação em categorias e, por fim, sua análise. O estudo foi aprovado por Comitê de Ética em Pesquisa. Resultados: A amostra final foi composta por 23 Acadêmicos de Enfermagem que aceitaram participar do estudo. O diálogo entre os participantes da pesquisa dos diversos períodos durante a abordagem e coleta de dados, tornou-se a pesquisa mais produtiva possibilitando elencar pontos relevantes sobre o processo de enfermagem sob a visão dos graduandos. Conclusão: O PE constitui um tema que ainda exprime uma grande lacuna entre a formação do entendimento e sua aplicação na prática diária dos acadêmicos. Assim, é fundamental a conscientização dos docentes para contribuir o conhecimento científico e a sagacidade técnica ao interesse, à dedicação e à sensibilidade fundamentais a uma assistência humanizada.
\end{abstract}

Palavras-Chave: Processo de Enfermagem, Acadêmicos, Enfermagem.

\begin{abstract}
Objective: To describe the meaning of the Nursing Process for the nursing students of a private college in Rondônia. Methods: This is a descriptive, exploratory study with a qualitative approach. The population studied corresponded to the academics from the fourth to the tenth period of the Nursing course. The research was performed through the application of a questionnaire containing 06 (six) open questions. The data obtained were analyzed by a method consisting of repeated readings of respondents' responses, their separation into categories and, finally, their analysis. The study was approved by the Research Ethics Committee. Results: The final sample consisted of 23 Nursing Academics who accepted to participate in the study. The dialogue between the participants of the research of the different periods during the approach and data collection became the most productive research making it possible to list relevant points about the nursing process from the perspective of the academic. Conclusion: The PE is a subject that still expresses a great gap between the formation of the understanding and its application in the daily practice of the academics. Thus, it is fundamental to raise the awareness of teachers to contribute scientific knowledge and technical sagacity to the interest, dedication and sensitivity fundamental to humanized assistance.
\end{abstract}

Keywords: Nursing Process, Academics, Nursing.

${ }^{1}$ União das Escolas Superiores de Rondônia (UNIRON), Porto Velho - RO. *E-mail: jaquelinemichele1985@hotmail.com

SUBMETIDO EM: 6/2019

ACEITO EM: 7/2019

PUBLICADO EM: 7/2019

REAS | Vol. Sup.29 | e1061 | DOI: https://doi.org/10.25248/reas.e1061.2019 Página 1 de 9 


\section{RESUMEN}

Objetivo: Describir el significado del Proceso de Enfermería para los académicos de Enfermería de una facultad privada de Rondônia. Métodos: Se trata de un estudio descriptivo, exploratorio con enfoque cualitativo. La población investigada correspondió a los académicos del cuarto al décimo período del curso de Enfermería. La investigación fue realizada a través de la aplicación de un cuestionario que contenía 06 (seis) preguntas abiertas. Los datos obtenidos fueron analizados por método que consiste en lecturas repetidas de las respuestas de los entrevistados, su separación en categorías y, finalmente, su análisis. El estudio fue aprobado por el Comité de Ética en Investigación. Resultados: La muestra final fue compuesta por 23 Académicos de Enfermería que aceptaron participar del estudio. El diálogo entre los participantes de la investigación de los diversos períodos durante el abordaje y recolección de datos, se convirtió en la investigación más productiva posibilitando enumerar puntos relevantes sobre el proceso de enfermería bajo la óptica del académico. Conclusión: EI PE es un tema que aún expresa una gran brecha entre la formación del entendimiento y su aplicación en la práctica diaria de los académicos. Por lo tanto, es fundamental aumentar la conciencia de los docentes para contribuir con el conocimiento científico y la sagacidad técnica al interés, la dedicación y la sensibilidad fundamentales para la asistencia humanizada.

Palabras clave: Proceso de Enfermería, Académicos, Enfermería.

\section{INTRODUÇÃO}

De acordo com a Resolução no 358/2009, do Conselho Federal de Enfermagem (COFEN), o Processo de Enfermagem (PE) caracteriza-se por ser um mecanismo metodológico que serve como orientação para os cuidados de enfermagem. Para tanto, o PE possui etapas que devem ser seguidas pelo enfermeiro, para que a assistência seja realizada de forma individualizada e integral. Assim, o PE divide-se nas seguintes etapas: coleta de dados, diagnósticos de enfermagem, planejamento, implementação e avaliação (COFEN, 2009).

Segundo Potter P e Perry AG (2013), o Processo de Enfermagem (PE) fundamenta-se em uma abordagem crítica e reflexiva sobre $o$ atendimento e prestação da assistência de enfermagem. O PE permite que o enfermeiro desenvolva e implemente um plano de cuidados individualizado e voltado para as necessidades do paciente.

A sua elaboração parte de um processo sistemático, contínuo e baseados em etapas subsequentes e cíclicas. Desta forma a visão do estudante de enfermagem é crucial para o desenvolvimento da aprendizagem e, consequentemente, na formação de futuros profissionais sob a ótica do processo de enfermagem padronizado, baseado em evidências e uma assistência de enfermagem de qualidade (RAMOS MA et al., 2015).

Esta compreensão sobre o processo de enfermagem, sob a ótica dos estudantes, é capaz, não somente de desenvolver o senso crítico e de aperfeiçoamento das práticas de enfermagem, como também contribui na formação de enfermeiros mais qualificados e com embasamento técnico científico (RAMOS MA et al., 2015).

O processo de enfermagem é um dos componentes fundamentais na formação dos profissionais enfermeiros, que são a arte e a ciência da enfermagem. A arte caracteriza-se pela relação enfermeiropaciente, que ocorre no momento da entrevista, na qual o enfermeiro coloca em prática a sua visão integral do paciente, por meio da investigação dos aspectos biopsicológicos, sociológicos, culturais e ambientais que podem influenciar no estado de saúde do paciente (BARROS ALBL, 2016).

Dentro do processo de enfermagem a coleta de dados objetivos, que podem ser observados por meio das técnicas propedêuticas e da anamnese. Contribuem para que com base nos achados e nos problemas detectados durante a coleta de dados, o enfermeiro poderá identificar os diagnósticos de enfermagem e traçar o plano de cuidados para o paciente (POTTER P e PERRY AG, 2013). 
O ensino na graduação em enfermagem caracteriza-se pela avaliação dos acadêmicos com base na realização das técnicas descritas na literatura. Esta forma de avaliação reforça o modelo biomédico, resultando em uma lacuna entre o futuro profissional e o paciente. O acadêmico, desta forma, passa a dar ênfase aos aspectos biológicos, desconsiderando as demais questões do ser humano que podem interferir diretamente no seu estado de saúde (AZEVEDO DM et al., 2013).

A assimilação do processo de enfermagem deve despertar no acadêmico o pensamento crítico reflexivo e lógico, a capacidade de discernimento, intuição, dentre outras habilidades necessárias para uma boa avaliação e delimitação de plano de cuidados. Entretanto, algumas instituições ainda não desenvolvem o ensino do processo de enfermagem em todas as suas etapas, dificultando a relação que o acadêmico deve fazer do conteúdo assimilado nas aulas teóricas com a prática em campo de estágio (LUZIA MF et al., 2013).

A importância da visão crítica quanto aos processos de enfermagem converge na formação participativa e reflexiva do estudante em sua própria formação. Uma das inovações no campo do ensino de enfermagem é o aprendizado pautado na participação da construção de sua própria educação (CARVALHO DP et al., 2015).

Uma das funções principais da faculdade de enfermagem não é somente a formação técnica dos seus estudantes, mas também a construção de profissionais conscientes e críticos quanto ao seu papel na sociedade, assim como o desenvolvimento de mentes que possam contribuir com o desenvolvimento da ciência e do cuidado em enfermagem, e é por meio da visão da perspectiva do estudante que os métodos de ensino são desenvolvidos (CARVALHO DP et al., 2015).

A presente pesquisa justifica-se pela necessidade de um maior aprofundamento sobre o significado e perspectivas dos acadêmicos da graduação em enfermagem sobre o processo de Enfermagem. Desta forma, o presente estudo levanta o seguinte problema: qual a ótica dos acadêmicos de enfermagem de uma faculdade privada sobre 0 processo de enfermagem? $O$ presente estudo tem por objetivo descrever 0 significado do Processo de Enfermagem dos acadêmicos de Enfermagem de uma faculdade privada de Rondônia.

\section{MÉTODOS}

Estudo descritivo, exploratório e qualitativa, mediante aplicação de questionário. A população pesquisada correspondeu aos acadêmicos do quarto ao décimo período do curso de Enfermagem da União das Escolas Superiores de Rondônia (UNIRON) que estejam cursando disciplinas que exigem carga horária prática no ambiente hospitalar. A amostra final foi composta por 23 Acadêmicos de Enfermagem, como critérios de inclusão, participou do estudo somente aqueles acadêmicos do quarto ao décimo período do curso de Enfermagem da UNIRON, devidamente matriculados na instituição, aprovados na disciplina de Bases Teórico-práticas de Enfermagem e que concordou em participar da pesquisa por meio da assinatura do Termo de Consentimento Livre e Esclarecido (TCLE). Foram excluídos do estudo aqueles discentes que ainda não cursaram a disciplina de Bases Teórico-práticas de Enfermagem na UNIRON e aqueles que cursaram a disciplina em outra instituição.

O estudo foi desenvolvido obedecendo ao estabelecido na Resolução n.. 466/2012/CNS/MS. Em virtude de se tratar de uma pesquisa com seres humanos, o estudo foi submetido ao Comitê de Ética em Pesquisa (CEP) e após parecer favorável, iniciou-se a coleta de dados. A pesquisa foi realizada através da aplicação de um questionário contendo 06 (seis) perguntas abertas, com duas para caracterização dos graduandos e outras relacionadas ao assunto abordado.

O questionário foi entregue aos participantes e no mesmo momento do término do preenchimento foram recolhidos. Após a sua assinatura do termo foi então entregue o questionário. $O$ presente estudo foi submetido à apreciação do CEP da UNINORTE, o qual expediu parecer favorável sob o número 3.234.562, em 30 de março de 2019. 


\section{RESULTADOS E DISCUSSÃO}

A amostra final foi composta por 23 Acadêmicos de Enfermagem que aceitaram participar do estudo. As informações principais referentes à caracterização dos acadêmicos de enfermagem que participaram da pesquisa estão dispostas no quadro abaixo. Observou-se que na Tabela 1 os acadêmicos são de diversos períodos do curso de enfermagem e a maioria com predominância do sexo feminino. Dos 23 graduandos de enfermagem que participaram do estudo, dez são do nono período, cinco do décimo, três do quarto, quatro do sétimo e um do quinto período, 20 são do sexo feminino, demonstrando mais uma vez a predominância do respectivo gênero.

Tabela 1 - Caracterização dos participantes da pesquisa. Porto Velho-RO, 2019.

\begin{tabular}{ccc}
\hline GRADUANDOS & GÊNERO & PERÍODO \\
\hline A1 & $\mathrm{F}$ & $4^{\circ}$ \\
A2 & $\mathrm{F}$ & $4^{\circ}$ \\
A3 & $\mathrm{F}$ & $4^{\circ}$ \\
A4 & $\mathrm{F}$ & $5^{\circ}$ \\
A5 & $\mathrm{F}$ & $5^{\circ}$ \\
A6 & $\mathrm{F}$ & $5^{\circ}$ \\
A7 & $\mathrm{F}$ & $7^{\circ}$ \\
A8 & $\mathrm{M}$ & $7^{\circ}$ \\
A9 & $\mathrm{F}$ & $7^{\circ}$ \\
A10 & $\mathrm{F}$ & $7^{\circ}$ \\
A11 & $\mathrm{F}$ & $9^{\circ}$ \\
A12 & $\mathrm{F}$ & $9^{\circ}$ \\
A13 & $\mathrm{M}$ & $9^{\circ}$ \\
A14 & $\mathrm{M}$ & $9^{\circ}$ \\
A15 & $\mathrm{F}$ & $9^{\circ}$ \\
A16 & $\mathrm{F}$ & $9^{\circ}$ \\
A17 & $\mathrm{F}$ & $9^{\circ}$ \\
A18 & $\mathrm{F}$ & $9^{\circ}$ \\
A19 & $\mathrm{F}$ & $9^{\circ}$ \\
A20 & $\mathrm{F}$ & $9^{\circ}$ \\
A21 & $\mathrm{F}$ & $9^{\circ}$ \\
A22 & $\mathrm{F}$ & $10^{\circ}$ \\
A23 & $\mathrm{F}$ & $10^{\circ}$ \\
\hline
\end{tabular}

Fonte: Barbosa JMS et al. 2019.

Em relação à predominância do sexo feminino em ambiente acadêmico, Pereira AL et al. (2015) destacaram em seu estudo realizado em oito hospitais de Santa Catarina, Florianópolis dos 50 acadêmicos de enfermagem pesquisados, 47 eram do sexo feminino. Silva ASR (2015) confirma relatando que em duas unidades de ensino do sul do Brasil, 120 dos 45 acadêmicos de enfermagem era com predominância do sexo feminino.

Constatou-se um predomínio de participantes do sexo feminino, sendo compatível com o resultado obtido por Pinto VA, Menezes EB e Villa NC (2014) que demonstrou na sua pesquisa que prevalece mais o sexo feminino.

Nesse sentido, Carrieri RB et al. (2013) afirma que a profissão de enfermagem é exercida, em sua maior parcela, por pessoas do sexo feminino tornando-se relevante quando se analisa os determinantes e a organização dessa prática social, e o estereótipo que cerca a experiência da mulher na sociedade.

A análise de conteúdo das respostas foi identificada como parte fundamental do processo de investigação como estratégia de compreensão, a partir do processo de enfermagem sob a ótica do acadêmico de uma faculdade privada.

Das respostas analisadas emergiram três categorias temáticas e ideias-chaves. Na primeira temática levantada, o Processo de Enfermagem e suas etapas, será discutida: A percepção dos graduandos de 
enfermagem quanto ao conceito e suas etapas do PE. A segunda temática abordará sobre as dificuldades do PE em cada fase, onde será levantada os obstáculos dos acadêmicos sobre o PE durante os estágios/práticas acadêmicas. Ao final da última temática levantando a importância do uso do PE como futuro enfermeiro, e nele discutirá sobre o interesse dos graduandos de enfermagem sobre a utilização do PE (Quadro 2).

Quadro 2 - Categorias temáticas e ideias-chaves levantadas, referente ao processo de enfermagem sob a ótica do acadêmico de uma faculdade privada. Porto Velho, Rondônia, 2019.

\begin{tabular}{|l|l|}
\hline \multicolumn{1}{|c|}{ Categorias temáticas } & \multicolumn{1}{|c|}{ Ideias-chaves } \\
\hline O Processo de Enfermagem e suas etapas & $\begin{array}{l}\text { A percepção dos graduandos de enfermagem quanto ao } \\
\text { conceito e suas etapas do PE. }\end{array}$ \\
\hline $\begin{array}{l}\text { As dificuldades do Graduando para } \\
\text { implementação do PE }\end{array}$ & $\begin{array}{l}\text { Os obstáculos dos acadêmicos sobre o PE durante os } \\
\text { estágios/práticas acadêmicas. }\end{array}$ \\
\hline $\begin{array}{l}\text { A importância do uso do PE para o futuro } \\
\text { enfermeiro }\end{array}$ & $\begin{array}{l}\text { O interesse dos graduandos de enfermagem sobre a } \\
\text { utilização do PE. }\end{array}$ \\
\hline
\end{tabular}

Fonte: Barbosa JMS et al. 2019.

\section{O Processo de enfermagem e suas etapas}

Nesta categoria temática, descreveremos o conceito do processo de enfermagem e quais são as etapas desse processo de enfermagem mediante as falas dos acadêmicos.

O Processo de Enfermagem tem como relação de exercícios, deveres, ações práticas e sistemáticas para a efetivação teórica ou prática de um serviço.

É um sistema de planejamento característico e direto para o atendimento das necessidades básicas dos pacientes, sendo um método extenso e vasto que abrange, além do estudo, o plano de cuidados e a conjuntura contextual geral em que este processo será ampliado (TAVARES AL, 2016).

De acordo com a descrição dos graduandos de enfermagem, na sua maior parte, definem o Processo de Enfermagem:

"O PE é a sistematização das ações do enfermeiro, um instrumento para auxiliar a detectar problemas na saúde do paciente e, desta forma guiá-lo na sua conduta". (A2)

"O Processo de enfermagem é dar a assistência de forma ampla, com o objetivo de orientar o cuidado e promover a qualidade da assistência prestada". (A6)

O diálogo entre os participantes da pesquisa dos diversos períodos durante a abordagem e coleta de dados, tornou-se a pesquisa mais produtiva possibilitando elencar pontos relevantes sobre o processo de enfermagem sob a ótica do acadêmico.

Segundo Soares AL (2015) o Processo de Enfermagem tenha sido apresentado por Wanda Horta, no ano 70 , nos dias de hoje pode-se notar que alguns graduando ainda têm pouco entendimento acerca da conceituação do PE na sua plenitude.

Ressalta-se que, o PE é de suma importância, pois proporciona uma assistência sistematizada, com uma visão holística direcionada ao cliente e não apenas à doença (SILVA VL, 2013).

Pedrosa AVR (2016) afirma que o PE é regido no Brasil como um processo que prepara o trabalho profissional, permitindo a implementação do PE, ferramenta metodológica que esclarece o cuidado profissional de enfermagem, dividido em cinco fases inter-relacionadas: coleta de dados, diagnóstico de enfermagem, planejamento, implementação e avaliação de enfermagem 
Observa-se mediante as falas que existe uma relevância no que diz respeito a conceituação do PE e quais seriam suas etapas a serem descritas como demonstram nas falas abaixo:

"O Processo de enfermagem é uma ferramenta para elaboração de uma assistência ao paciente realizada pelo enfermeiro". (A12)

"É o método que direciona e organiza de forma sistematizada o trabalho do enfermeiro, as etapas são cinco: histórico de enfermagem, diagnóstico, implementação, planejamento e avaliação". (A23)

Moraes ACFA (2016) complementa que o PE é imprescindível para orientar a assistência de enfermagem, onde o profissional que o utiliza como instrumento norteador será apto de aperfeiçoar habilidades cognitivas e psicomotoras para relacionar teoria e prática, associando entendimentos multidisciplinares e determinando relações de trabalho mais intensas e prolíficos, visando uma melhor qualidade no atendimento ao cliente.

Na pesquisa Menossi MFL et al. (2015), descrevem que o processo de enfermagem é percebido pelos acadêmicos como um instrumento que tem a função de organizar a assistência de Enfermagem, remetendo a uma função técnica gerencial das ações de Enfermagem.

Sobre essa ótica, o processo de trabalho do enfermeiro teve um avanço com a utilização dos conhecimentos da área de administração, pois estes influenciaram na sistematização dos cuidados de enfermagem adotada nas instituições hospitalares, contribuindo para a valorização das ações, maior visibilidade e ampliação dos campos de atuação (MACHADO LCN, 2016).

Outro achado foi o conhecimento de algumas etapas do processo de enfermagem por parte dos graduandos de enfermagem.

\section{As dificuldades do graduando para implementação do PE}

No que tange as dificuldades dos graduandos de acordo com as fases do PE durante os estágios/práticas acadêmicas, a maioria dos graduandos refere ter aprendido e executado as etapas do PE na graduação, mas as dificuldades na prática são significativas, retratando a insuficiência do preparo teórico e prático do acadêmico para a implementação do instrumento quando concluem a graduação, como se observa nas falas dos participantes a seguir:

"A dificuldade tenho um pouco na evolução de enfermagem, pois para os professores cada um tem uma maneira de querer uma evolução perfeita e diferente das outras". (A17)

"Tenho dificuldade com os diagnósticos de enfermagem é muito complicado a elaboração." (A22)

"Tenho dificuldade em fazer o diagnóstico de enfermagem, pois ainda tenho uma certa dificuldade em usar o livro Nanda". (A15)

Andrade JS e Vieira MJ (2015) corroboram com as falas supracitadas dizendo que a evolução propicia ao acadêmico a possibilidade da prestação da assistência individual, relatando nas necessidades humanas básicas, bem como destina-se a tomada de decisão em várias situações vividas pelo enfermeiro como gerenciador da equipe de enfermagem.

É importante frisar que durante a graduação, os discentes são ensinados a realizar cada fase do PE e a utilizar os sistemas de classificações de enfermagem e, a partir da junção de conhecimentos com outras disciplinas do curso, eles põem em prática a sequência utilizada na assistência (PEDROSA AVR, 2016).

Outra dificuldade que Farias AL (2016) aborda é sobre o diagnóstico de enfermagem, isso se deve ao fato de que as aulas práticas não foram suficientes para ampliação dos conhecimentos sobre as fases do PE. Com isso passam a fragmentar os cuidados e os problemas do paciente, deixando de vê-lo como um todo, e muitas vezes adotando cuidados que não tem relação com os problemas encontrados. 
Nessa perspectiva de Pinho AMP (2015), conclui que apesar dos esforços, ainda existem dificuldades na implementação do PE, relacionadas, por exemplo, ao processo de ensino-aprendizagem, como a falta de aula prática para o desenvolvimento dessa ferramenta, fazendo com que muitos profissionais de enfermagem deixem de sistematizar sua assistência.

Algumas pesquisas apontam que a deficiência no conhecimento e na aplicação do PE durante a vida acadêmica é um problema significativo, visto que é na academia que se prepara o futuro profissional para o mercado de trabalho (MOURA MD, 2015).

Tais problemas tornam o processo de enfermagem mais difícil de ser executada de uma maneira satisfatória, os quais podem analisar a partir das falas a seguir:

"As dificuldades encontradas na fase do PE são durante o planejamento das intervenções" (A4).

"A falta de profissionais suficientes para realizar cada etapa em cada paciente" (A16).

Diante dos resultados apresentados, Peres MH (2014) percebeu-se que as dificuldades dos acadêmicos de enfermagem na aplicação do Processo de Enfermagem como instrumento de trabalho se devem, em parte, à formação acadêmica dos profissionais enfermeiros, que não está voltada para a valorização da aplicabilidade do PE e suas respectivas etapas.

Vieira MQ (2014) evidencia em sua pesquisa que outras dificuldades observadas na aplicabilidade do PE reafirmam as já mencionadas no presente estudo, dentre elas temos a deficiência dos discentes e profissionais na realização do exame físico, na padronização do ensino pelos professores, além da falta de conhecimento sobre fisiopatologia.

Para tanto, as consequências da não aplicação do Processo de Enfermagem referem-se aos cuidados e aos problemas do cliente, deixando de vê-lo como um todo e, muitas vezes, adotando cuidados que não têm relação com os problemas encontrados (PINHO AMP, 2015).

A falta de profissionais de saúde suficientes para realizar o processo de enfermagem, torna-se barreira para a sua adesão à execução deste método assistencial nas instituições de saúde.

Quando o realizam sem o necessário conhecimento, o fazem apenas para o cumprimento de tarefa institucional, não havendo a conscientização coletiva da importância deste processo para a sua atuação, como profissional da saúde com responsabilidade social (DOLERES RC, 2016).

\section{A importância do uso do PE para o futuro enfermeiro}

Na pesquisa de Tavares AL (2016), constata-se que a maior parte dos graduandos acham fundamental a utilização do PE, alguns porque concordam que essa ferramenta simplifica os cuidados de enfermagem, e outros porque pode-se presenciar a evolução do paciente, melhorando sua qualidade de vida e gerando 0 autocuidado.

O processo de enfermagem é deste modo, de fundamental importância para a atuação da enfermagem, tendo em vista que permite o pensamento crítico para elaboração de diagnósticos que atenderam as necessidades humanas básicas afetadas e consequentemente uma assistência de enfermagem de qualidade ao cliente (SILVA VS, 2013).

Como se observa nos depoimentos:

"O uso do processo de enfermagem é muito importante para o exercício da enfermagem, pois ela guia o profissional da enfermagem no atendimento ao paciente" (A18).

"É de grande importância o uso desse processo, pois faz com que a equipe de enfermagem preste uma assistência adequada aos pacientes e ajuda muito o setor e a equipe" (A3). 
Assim sendo, as etapas do processo de enfermagem devem ser respeitadas e seguidas coerentemente para que ocorram intervenções e resultados esperados e assim condições de obter eficácia no tratamento do cliente (PADILHA DA, 2015).

George RWL (2013) descreve que, a partir do PE, a prática é realizada de maneira sistemática e organizada, constituindo num instrumento de metodologia de enfermagem, favorecendo e analisando consequências.

Nota-se que para alguns acadêmicos de enfermagem, o PE faz-se notável por dar seguimento a assistência de enfermagem como um roteiro, voltado a prática, segundo observa-se nos relatos dos participantes a seguir:

"É um instrumento primordial para elaboração do diagnóstico de enfermagem para que os enfermeiros podem empregar suas ações de acordo com as Necessidades humanas do paciente". (A1)

"O Processo de Enfermagem é muito importante para se prestar uma assistência de qualidade ao paciente". (A14)

Conforme George RWL (2013), os graduandos em enfermagem que usam o processo estão compreendendo a exercer como profissionais em sua execução. Os mesmos devem adaptar-se com o processo e tornando-se habilidosos em sua utilização, no qual auxiliará como suporte no desenvolvimento da sua assistência de enfermagem.

De acordo com Waldow EG (2015), percebe-se a relevância da utilização do PE, mas é evidente a determinação por parte dos enfermeiros em concordar e empregar esse mecanismo científico de trabalho, as causas básicas são os mesmos, ou seja, duração, números de pessoas compondo a equipe e por ser considerada uma atividade teórica, sem aplicabilidade prática.

Os graduandos de enfermagem estão inseguros na sua aplicação na prática devido à base disciplinar, cabendo às instituições de enfermagem a promoção de uma extensa discussão sobre o tema, a fim de definir o embasamento teórico que fundamentara o PE, ou seja, conceituação por uma ou mais fundamentos na enfermagem ou marco intelecto, e conceituar a metodologia a ser utilizada no ensino do $\mathrm{PE}$, conforme os relatos a seguir:

"É importante para o desenvolvimento de uma assistência de enfermagem com qualidade e eficaz para o paciente". (A19)

"Suma importância se dá por ser o pilar essencial no cuidado e manutenção do cuidado com o paciente". (A7)

Para os graduandos, essa falta de entendimento do PE surgiu na adaptação e carência de interesse dos acadêmicos em argumentar, investigar e analisar os informes recebidas da Faculdade, quando precisavam pesquisar e enriquecer seu conhecimento através dos embasamentos teóricos (PADILHA DA, 2015).

Para Pinho AMP (2015) o uso do PE é visto pelos maior parte dos graduandos como uma peça essencial para a enfermagem. O PE facilita a assistência por sistematizar as ações de enfermagem.

Azevedo DM (2016) acredita que o PE é um modelo assistencial específico, que evidencia a posição e objetivos da enfermagem de acordo com seus embasamentos científicos. Assim como os graduando, os enfermeiros têm interesse em usá-lo.

\section{CONCLUSÃO}

O PE constitui um tema que ainda exprime uma grande lacuna entre a formação do entendimento e sua aplicação na prática diária dos acadêmicos. Assim, é fundamental a conscientização dos docentes para contribuir o conhecimento científico e a sagacidade técnica ao interesse, à dedicação e à sensibilidade fundamentais a uma assistência humanizada. 


\section{REFERÊNCIAS}

1. AZEVEDO DM. Da academia à realidade: uma reflexão acerca da prática do exame físico nos serviços de saúde. Saúde Transform. Soc., Florianópolis, v. 4, n. 4, p. 106-110. 2016.

2. ANDRADE JS de, VIEIRA MJ. Prática assistencial de enfermagem: problemas, perspectivas e necessidade de sistematização. Rev. bras. enferm., Brasília, v. 58, n. 3, p. 261-265, June 2015.

3. BARROS ALBL de. Anamnese e exame físico: avaliação diagnostica de enfermagem no adulto. 3. ed. Porto Alegre: Artmed. 2016. 362p.

4. CARVALHO DP et al. Teoria da aprendizagem significativa como proposta para inovação no ensino de enfermagem: experiência dos estudantes. Revista de Enfermagem da UFSM, [S.I.], v. 5, n. 1, p. 186 - 192 , abr. 2015.

5. CARRIERI RB et al. Desenvolvimento de um website educacional para o ensino do processo de enfermagem em cardiologia. Revista Enfermagem em Foco, v. 8, n. 2. 2013.

6. CONSELHO FEDERAL DE ENFERMAGEM (COFEN). Resolução COFEN-358/2009. Dispõe sobre a Sistematização da Assistência de Enfermagem e a implementação do Processo de Enfermagem em ambientes, públicos ou privados, em que ocorre o cuidado profissional de Enfermagem, e dá outras providências.

7. DOLERES RC. Exame físico na percepção dos discentes do curso de Enfermagem: da teoria a pratica assistencial. FIEP Bulletin On-line. v. 85. 2016.

8. FARIAS AI. Qualidade na assistência de enfermagem hospitalar: visão de alunos de graduação. Revista Gaúcha Enfermagem, Porto Alegre, v.21, n. 3, p. 529-535, set, 2016.

9. GEORGE RWL. Sistematização da assistência de enfermagem na perspectiva dos enfermeiros: uma abordagem metodológica na teoria fundamentada. Revista Gaúcha Enfermagem, v. 33, n. 3, p.174-18, 2013.

10. LUZIA MF et al. O ensino das etapas do processo de enfermagem: revisão integrativa. Rev. Enferm, UPE online, esp. 7, p. 6678-87, nov., 2013.

11. MACHADO LCN. Saturação teórica em pesquisa qualitativa: relato de experiencia na entrevista com escolares. Revista Brasileira de Enfermagem. v. 1, n. 71, 2016.

12. MENOSI MFL et al. Percepções de estudantes sobre o exame físico na pratica clínica do enfermeiro. Rev. Rene. V. 17, n. 2. P. 268-77, 2015.

13. MORAES ACFA. Exame físico cardiorrespiratório: conhecimento de estudantes de enfermagem. J. Res.: fundam. Care. Online. v. 1, n. 7. P. 1967-1974. 2016.

14. MOURA MD et al. Contribuições da monitoria em semiologia e semiotécnica para a formação do enfermeiro: relato de experiência. UDESC em Ação. v. 7, n. 1. 2015.

15. PADILHA DA enfermagem enquanto disciplina, profissão e trabalho. Revista Brasileira de Enfermagem, Brasília, v.62, n. 5, p. 739-744, set-out. 2015.

16. PERES MHO ensino da sistematização da assistência de enfermagem na visão do docente. Revista Nursing, v. 10, n. 8, p. 141-146, mar., 2014.

17. PEREIRA RS et al. Sistematização da assistência de enfermagem em unidade de reabilitação segundo o modelo conceitual de Horta. Rev. bras. enferm., Brasília, v. 59, n. 4, p. 556-559, 2015.

18. PINHO AMP. Sistematização da Assistência de Enfermagem: Guia Prático. 2. ed. Rio de Janeiro: Guanabara Koogan, 2015. 52p.

19. PINTO VA, MENEZES EB, VILLA NC. Sistematização da assistência de enfermagem: problemas identificados pelos enfermeiros. Revista Técnica Nursing, São Paulo, v. 18, n. 4, p. 28-34, 2014.

20. POTTER P, PERRY AG. Fundamentos de enfermagem. Rio de Janeiro: Elsevier, 2013.

21. PEDROSA AVR. Aplicação do processo de enfermagem: promoção do cuidado colaborativo. 5 ed. Porto Alegre: Artes Médicas; 2016. 124p.

22. RAMOS MA. et al. Satisfação com a experiencia acadêmica entre estudantes de graduação em enfermagem. Texto \& Contexto Enfermagem. 2015,

23. SILVA VS et al. Utilização do processo de enfermagem e as dificuldades encontradas por enfermeiros. Cogitare Enfermagem. v. 2, n. 18, 2013.

24. SILVA IEF. Sistematização da assistência de enfermagem: percepção dos enfermeiros de um hospital filantrópico. Acta Scientiarum. Health Science. 2015.

25. SOARES J. Dificuldades na implantação da sistematização da assistência de enfermagem - SAE: uma revisão teórica. Cadernos da Escola de Saúde, Curitiba; 2015.

26. TAVARES AL. Sistematização da assistência: aproximando o saber acadêmico, o saber fazer e o legislar em saúde. Acta paul. enfer. v.1, n.3, São Paulo. 2016.

27. VIEIRA MQ. A implementação da sistematização da assistência de enfermagem no serviço de saúde hospitalar do Brasil. Texto \& contexto enferm. São Paulo. 2014.

28. WALDOW EG. O desafio de implantar a sistematização da assistência de enfermagem sob a ótica de discentes. Esc. Anna Nery Rev. Enferm, v.2, n.3. São Paulo. 2015. 